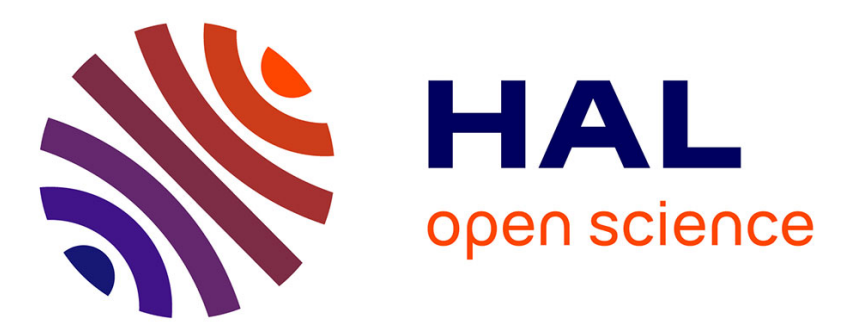

\title{
The role of trait anxiety on static balance performance in control and anxiogenic situations
}

Jean-Philippe Hainaut, Grégory Caillet, Francis G. Lestienne, Benoît Bolmont

\section{To cite this version:}

Jean-Philippe Hainaut, Grégory Caillet, Francis G. Lestienne, Benoît Bolmont. The role of trait anxiety on static balance performance in control and anxiogenic situations. Gait \& Posture, 2011, 33

(4), pp.604-608. 10.1016/j.gaitpost.2011.01.017 . hal-01345186

\section{HAL Id: hal-01345186 \\ https://hal.univ-lorraine.fr/hal-01345186}

Submitted on 9 Oct 2019

HAL is a multi-disciplinary open access archive for the deposit and dissemination of scientific research documents, whether they are published or not. The documents may come from teaching and research institutions in France or abroad, or from public or private research centers.
L'archive ouverte pluridisciplinaire HAL, est destinée au dépôt et à la diffusion de documents scientifiques de niveau recherche, publiés ou non, émanant des établissements d'enseignement et de recherche français ou étrangers, des laboratoires publics ou privés. 


\title{
The role of trait anxiety on static balance performance in control and anxiogenic situations
}

\author{
Jean-Philippe Hainaut ${ }^{\mathrm{a}, \mathrm{b}}$, Grégory Caillet ${ }^{\mathrm{a}}$, Francis G. Lestienne ${ }^{\mathrm{b}}$, Benoît Bolmont ${ }^{\mathrm{a}, \mathrm{b}, *}$ \\ a Université Paul Verlaine-Metz, UFR SciFa, Dept STAPS, LASC (EA 3467) Emotions-Actions, Campus-Bridoux, Avenue Général Delestraint, 57070 Metz-Borny, France \\ ${ }^{\mathrm{b}}$ ERT 2002 «RAPSODIE», 2 Bd du Maréchal Juin 14032 CAEN, France
}

A R T I C L E I N F O

\section{Article history:}

Received 28 July 2010

Received in revised form 24 December 2010

Accepted 25 January 2011

\section{Keywords:}

Static balance

State anxiety

Trait anxiety

Personality

Visual dependence

\section{Introduction}

The human balance control system involves complex motor performance that depends on sensory perception, central cognitive processing and motor response performance. Several studies have used posturographic parameters as revealed by centre of foot pressure (CFP) displacements in order to investigate balance control [1]. As far as static posturography is concerned, low values for both sway path length (SPL: distance in mm covered by the CFP displacement) and sway area parameters (SA: surface in $\mathrm{mm}^{2}$ covered by the CFP displacement) reflect efficient balance control. While the SPL parameter is closely linked to sway velocity, the SA parameter is considered to be the most representative parameter regarding precision in balance [2]. Postural stabilization depends on visual, vestibular and somatosensory inputs. The importance of vision in postural stabilization is well known. Although the SA parameter with eyes open depends on distance to target fixation (SA decreases when the distance is shortened) [3,4], classically, the SA parameter is larger with eyes closed (EC) than eyes open (EO). Indeed, the lack of visual input decreases postural performances.

\footnotetext{
* Corresponding author at: Université Paul Verlaine-Metz, UFR SciFa, Dept STAPS, LASC (EA 3467) Emotions-Actions, Campus-Bridoux, Avenue Général Delestraint 57070 Metz-Borny, France. Tel.: +33 387378 671; fax: +33 387378603 .

E-mail address: bolmont@univ-metz.fr (B. Bolmont).
}

When their eyes are closed, subjects compensate for the lack of visual input by an ankle stiffness strategy, in order to enhance somatosensory perception [5]. This sensory re-weighting strategy could help subjects to maintain precision of balance when vision is absent.

Moreover, studies have shown that balance performance could depend on psychological state, such as state anxiety [6-10]. The fact that patients suffering from different pathological anxiety disorders are frequently confronted with postural instability has been highlighted $[11,12]$. This association between anxiety and balance disorders is not restricted to clinical samples and even occurs in healthy subjects $[13,14]$. State anxiety in healthy subjects could be considered to be a transitory state of either apprehension or arousal, resulting from the anticipation of an actual or nonactual threatening event, respectively. It has been shown that state anxiety caused by actual threat of a fall could lead a healthy subject to develop a postural stiffness strategy in order to avoid this fall; this strategy is characterized by a smaller amplitude and higher frequency in CFP displacements $[6,8,15,16]$. In contrast, few studies have investigated the effect of state anxiety not directed toward potential postural threat $[7,9,10]$ on static balance performance. When vision is available, state anxiety could influence interactions of visual input with vestibular and somatosensory inputs $[9,10]$. Another study on this issue, one which used a computer-controlled movable platform, has also shown that state anxiety and adverse mood changes could affect 
the subjects' ability to use inputs from the vestibular system in order to maintain balance [7]. These studies suggest that state anxiety could influence one or more sensory inputs underlying static balance control. In previous studies using a sensorimotor task, it has been shown that individuals possessing different trait anxiety levels preferentially process some sensory modalities in an anxiogenic condition $[17,18]$. It should be noted that state anxiety is transitory, while trait anxiety is a personality trait conceived as dispositions to perceive and to behave in specific ways, particularly when state anxiety increases [19]. In addition to the posturographic parameters, state and trait anxiety could be helpful in assessing individual sensory strategies used to maintain static balance. Thus, in agreement with these previous results and with Spielberger's concept [19], we hypothesize that the weight of sensory inputs involved in balance control could be differently affected by state anxiety, depending on trait anxiety level.

Consequently, the present study was designed to determine the influence of state anxiety on static balance performance with EO and EC in two groups of healthy human subjects with contrasting trait anxiety levels. Balance performance is assessed in a control condition and an anxiogenic condition induced by the Stroop Color-Word Test with interference, combined with video recording $[17,18,20,21]$, which is a moderate cognitive stressor.

\section{Materials and methods}

\subsection{Subjects}

Subject selection included a cohort of 56 participants (26 females and 30 males) studying for a degree at the University who volunteered for this experiment (mean age: $19.8 \pm 1.5$ ). All subjects were healthy individuals with no history of psychiatric, orthopaedic or neurological disorders. None of them took medication that could alter postural control or cognitive processing. Each participant provided full, informed consent according to the declaration of Helsinki before data collection. Trait anxiety was assessed using the Spielberger's State-Trait Anxiety Inventory (STAI [22]).

From this cohort, two groups were constituted by selecting the subjects whose scores on trait anxiety were at the extremes of the distribution (the 9 women and 9 men with higher trait anxiety and the 9 women and 9 men with lower trait anxiety). According to Spielberger's classification, the 18 subjects with higher scores formed the 'Intermediate Trait Anxiety' (ITA) group (score range: 40-62; mean score: $48.8 \pm 6$; male $(n=9)$ mean score: $47.1 \pm 3.9$; female $(n=9)$ mean score: $50.5 \pm 7.4)$, and the 18 subjects with lower scores formed the Very Low Trait Anxiety (VLTA) group (score range: $23-35$; mean score: $29.6 \pm 3.5$; male $(n=9)$ mean score: $29.8 \pm 2$; female $(n=9)$ mean score: $29.4 \pm 4.6)$.

\subsection{Assessment of balance}

Stabilometric parameters were obtained using a static force platform fitted with three strain gauges (with Winposture Software; Medicapteurs, France), with a sampling frequency of $40 \mathrm{~Hz}$. Subjects stood barefoot on the platform with their feet angled 30 relative to each other. They were asked to stand with their arms at their sides, looking at a target placed at eye level about $2 \mathrm{~m}$ in front of them in a quiet room. Each trial lasted $25.6 \mathrm{~s}$ (this duration allows 1024 measurements in $40 \mathrm{~Hz}$ ) because state anxiety induced by the anxiogenic condition is moderate and transitory. The SA and the SPL were determined according to displacements of CFP. The CFP parameters were corrected for subject height.

\subsection{Assessment of state and trait anxiety}

State and trait anxiety were assessed using the STAI Y1 and Y2 forms [22], respectively. These two self-evaluated questionnaires consist of 20 items, each scored on a four-point scale. State anxiety is considered to be a temporary state in response to actual or non-actual threats, whereas trait anxiety is a relatively stable personality trait that reflects dispositions to develop state anxiety [19].

\subsection{Control and anxiogenic conditions}

In the anxiogenic condition, moderate state anxiety was induced by using the Stroop Color Word Test with interference [17,18,20,21]. This test consists of color words printed in incongruent colors. Each subject was asked to say as quickly as possible and in the sequence presented, the color of the ink used to print a color name, not the color designated by the word. Hesitations or errors were indicated by means of a strident sound. Moreover, as used in procedures that simulate public performance, the subjects' performance was video recorded and shown on a TV screen placed in the visual field of the subject (below the fixation target) in order to increase state anxiety [20]. Furthermore, in order to maintain the anxiogenic characteristics of the task, subjects did not receive any training. This procedural decision was designed to avoid habituation effects that could lead the subjects to better cope with the situation.

In the control condition, the subjects were asked to perform a Stroop Color Wordlike Test with no interference that consisted of color words printed in congruent colors. The subjects were asked to name the color that appeared in the sequence at a normal reading tempo. Errors were not indicated and the subjects were not video recorded while performing the test.

In each condition, the subjects were asked to stand upright on the platform while they performed the color test. The color tests were located in the same place as the fixation target and were removed immediately after completion.

\subsection{Behavioral procedure}

The experimental procedure is presented in Fig. 1. All subjects were asked to perform the control condition first, and were then exposed to the anxiogenic condition. Throughout the condition (whether control or anxiogenic), subjects stood upright on the platform and alternately performed color tests and balance assessments. In this way, in each condition, the subjects had (1) to execute the color test within a $40 \mathrm{~s}$ period (the time to read about 50 words), (2) to stand upright for balance assessment with open eyes ( $25.6 \mathrm{~s}),(3)$ to execute the color test during a $40 \mathrm{~s}$ period and immediately after, to close their eyes, (4) to stand upright for balance assessment with closed eyes (25.6 s). All these tasks were performed continuously (i.e. with no transition period). Instructions were given before the beginning of each condition (control and anxiogenic). The subjects were asked to look at the fixation target and to stand as still as possible during the balance assessment. At the end of the color tests, the subjects were asked to look at the fixation target during the EO test or to look at the fixation target and then to close their eyes during the EC test. The order of the tasks (EO and EC) was specifically determined in order to correspond to a standardized measure of posturographic assessment. The control condition was conducted before the anxiogenic condition, so that the subject would not be disturbed by the induced anxiety state. Before the beginning of the experiment, $60 \mathrm{~s}$ practice trials were performed under $\mathrm{EO}$ and EC conditions to familiarize participants with the tests. Rest periods of 5 min were given before the experimental session and between the control and anxiogenic conditions.

The subjects had to complete a psychometric questionnaire for state anxiety before the experiment, after the control condition and after the anxiogenic condition. After each condition, the questionnaires were immediately completed according to the subjects' general feelings during the balance performances (including EO and EC tests).

\subsection{Data analyses}

A two-way ANOVA was applied to state anxiety with condition (repeated measures: baseline, control condition, anxiogenic condition) and group (ITA, VLTA subjects) as factors. Posturographic performances (SA and SPL) were analysed using three-way ANOVAs with conditions (repeated measures: control, anxiogenic), vision (repeated measures: EO, EC) and group (ITA, VLTA subjects) as factors. Following the occurrence of a significant interaction, two-way ANOVAs with

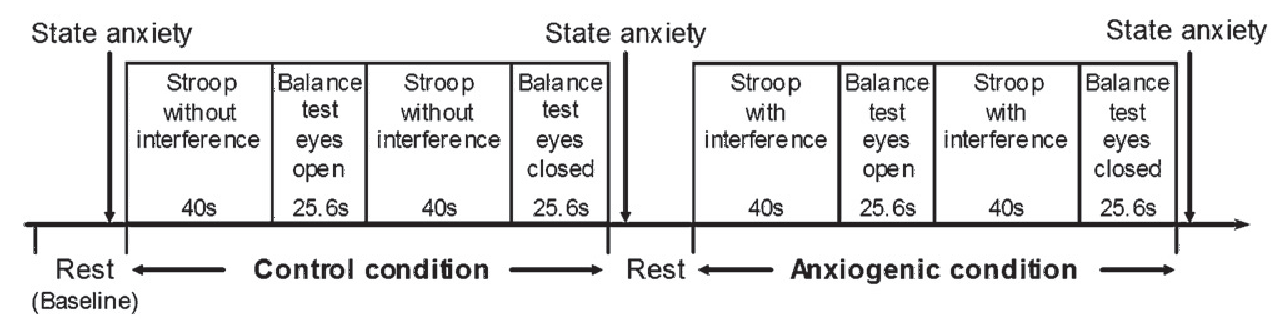

Fig. 1. Experimental procedure of the experiment. Psychometric questionnaires were completed during the first rest period (baseline) and after the control and the anxiogenic conditions. All subjects performed the control condition first and then were exposed to the anxiogenic condition. 
conditions (repeated measures: control, anxiogenic) and group (ITA, VLTA subjects) as factors, were used to analyse posturographic performances with open eyes and with closed eyes. Moreover, for the control condition, two-way ANOVAs with vision (repeated measures: EO, EC) and group (ITA, VLTA subjects) as factors, were used to analyse SA and SPL parameters. Following the occurrence of a significant $F$-value Tukey's test was used to perform post hoc comparisons in order to determine pairwise differences. Statistical significance was accepted for $p$ values lower than 0.05 .

\section{Results}

\subsection{Psychological parameters}

Changes in state anxiety are shown in Table 1 . Statistical analyses on state anxiety as measured by the STAI showed main effects for condition $\left(F_{(2,68)}=12.25 ; p<0.0001\right)$ and group $\left(F_{(1,34)}=14.35 ; p<0.001\right)$ factors, but no interaction between these factors was found. Post hoc analyses carried out on both groups showed a significantly higher level of anxiety in the anxiogenic condition as compared to the baseline $(p<0.01)$ and the control $(p<0.001)$ period.

\subsection{Posturographic parameters}

Changes in balance performances are shown in Fig. 2. The results of the three-way ANOVAs showed significant three-way interactions for the SA $\left(F_{(1,34)}=4.89 ; p<0.05\right)$ and the SPL $\left(F_{(1,34)}=14.04 ; p<0.001\right)$ parameters. Therefore, two-way ANOVAs were used to analyse posturographic performances with open eyes and with closed eyes. Analyses with open eyes revealed a main effect for the condition factor both for $S A\left(F_{(1,34)}=33.20\right.$; $p<0.0001)$ and for SPL $\left(F_{(1.34)}=17.81 ; p<0.001\right)$. Post hoc analyses showed that for both groups, SA $(p<0.001)$ and SPL $(p<0.001)$ increased in the anxiogenic condition. Conversely, analyses of posturographic performances with closed eyes showed significant interactions between condition and group factors for SA $\left(F_{(1,34)}=14.84 ; p<0.001\right)$ and for SPL $\left(F_{(1,34)}=13.55 ; p<0.001\right)$. With regards to the SA parameter, a significant increase during anxiogenic condition was found for the VLTA group $(p<0.01)$ but not for the ITA group. As for the SPL parameter, a significant increase during the anxiogenic condition was found for the VLTA group $(p<0.01)$ but not for the ITA group. No inter-group difference was observed either in the control condition, or in the anxiogenic condition.

Differences in balance performances between eyes-open and eyes-closed tests in the control condition are shown in Fig. 3. Analyses of posturographic performances in the control condition revealed a significant interaction between vision and group factors for SA $\left(F_{(1,34)}=5.12 ; p<0.05\right)$ but not for SPL. Post hoc comparisons between SA with open eyes and SA with closed eyes only showed a difference for the ITA group: the SA parameter was significantly higher when the eyes were closed $(p<0.01)$. With regards to the SPL parameter, a main effect was found for the vision factor $\left(F_{(1,34)}=53.41 ; p<0.0001\right)$. Post hoc analyses showed a higher SPL with EC than with EO $(p<0.001)$ in both groups.

It should be noted that initially, 'sex' was included in the ANOVAs as a factor, but no interaction or significant effect of sex was observed on data either for the psychological parameters or the posturographic performances. Therefore this factor was excluded from the analyses. Furthermore, no significant difference for the number of errors during the performance of the Stroop test was found between the VLTA and the ITA group.

\section{Discussion}

The aim of this study was to determine whether sensory inputs involved in static balance performance are affected differently by moderate state anxiety according to trait anxiety level. As shown in previous studies, the present results confirm that the video recorded Stroop Color-Word Test with interference, which is a cognitive stressor, induces a moderate but significant increase in state anxiety, whatever the subjects' trait anxiety $[17,18,20]$. The main finding of this study is that a moderate state anxiety increases the SA and SPL balance parameters (i.e., larger and faster body sways) for both VLTA and ITA groups when visual input is available. In contrast, with EC, the anxiogenic condition affects balance performance differently according to the level of trait anxiety.

When visual input is available, our results show that whatever the subjects' trait anxiety level, SA and the SPL parameters increase in the anxiogenic condition. Studies have shown that state anxiety could modify multisensory integration by modulating the processing of visual input $[9,10]$ and/or by exerting direct effects on vestibular functioning [23-25], provoking larger and faster body sways, which increase the SA and SPL parameters. In the present study, similar effects may have occurred. In addition, the somatosensory system could also be influenced by state anxiety. Previous studies showed that fear of falling induces anxiety and leads subjects to adopt a postural stiffness strategy (increased cocontraction of ankle joint agonist/antagonist muscle pairs) characterized by smaller amplitude and higher frequency in CFP displacements $[6,8,15,16]$. In contrast, in the present study, state anxiety that is not related to a potential postural threat may have led subjects to adopt different behavior without muscular cocontraction to fix posture. However, muscular tension associated with state anxiety $[21,26]$ may have increased motor activity, which in turn increased both SA and SPL parameters. Finally, when all sensory inputs are available, state anxiety may modify the processing or integration of visual, vestibular and/or somatosensory inputs, whatever the trait anxiety level. In the present study, this led subjects to perform larger and faster body sways.

When vision is not available, results have shown a different effect of the anxiogenic condition on static balance performance according to the level of trait anxiety. Previous studies on this issue

Table 1

Trait anxiety and state anxiety for the two groups measured in the first rest period (baseline), in the control condition and in the anxiogenic condition.

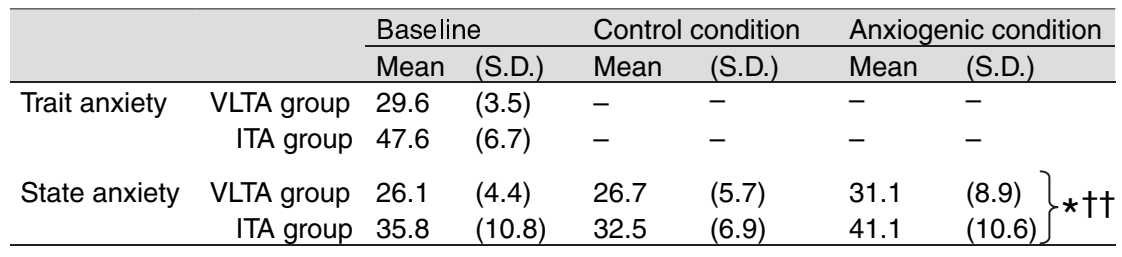

State and trait anxiety were evaluated by the STAI [22].

*Significantly different from baseline $(p<0.01)$.

${ }^{\dagger}$ Significantly different from control condition $(p<0.001)$. 
Eyes open
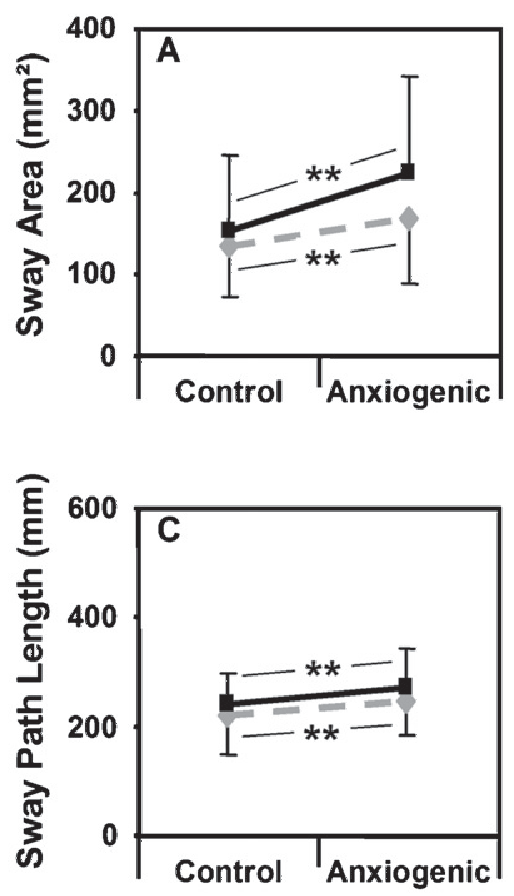

$\rightarrow$ VLTA group
Eyes closed
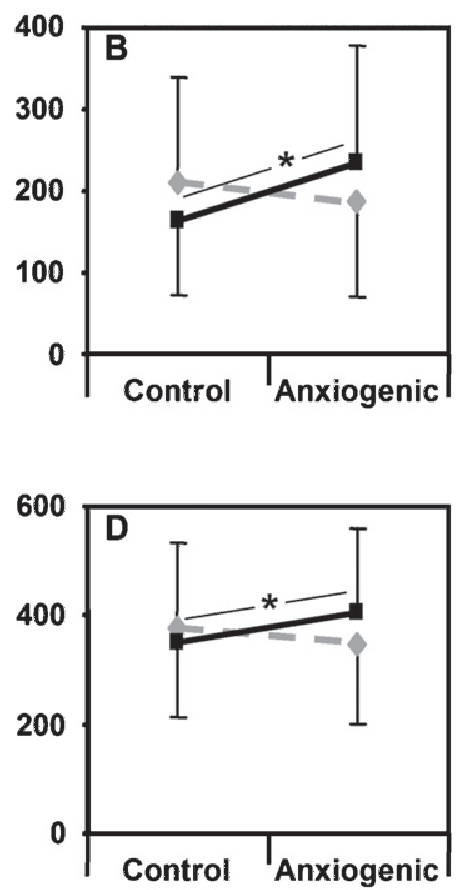

ITA group

Fig. 2. Differences in balance performances with eyes open (A, C) and eyes closed (B, D) for the sway area ( $m e a n$ in $\left.\mathrm{mm}^{2} ; A, B\right)$ and the sway path length (mean in mm; C, D) between the control condition and the anxiogenic condition for the very low trait anxiety group and the normal trait anxiety group. ${ }^{*} p<0.01$, ${ }^{* *} p<0.001$, significantly different from the control condition.

did not show any influence of state anxiety in the EC condition $[9,10]$, but none of these took the level of trait anxiety into account. In the present study, the VLTA group but not the ITA group increased the SA parameter and the SPL parameter in the anxiogenic condition. These results may provide initial insight into inter-individual trait anxiety differences in managing static balance without visual input.

Similarly to the test performed with visual input, VLTA subjects have larger and faster body sways in the anxiogenic condition when vision is absent. In agreement with studies that proposed a relationship between sensory inputs and psychological states
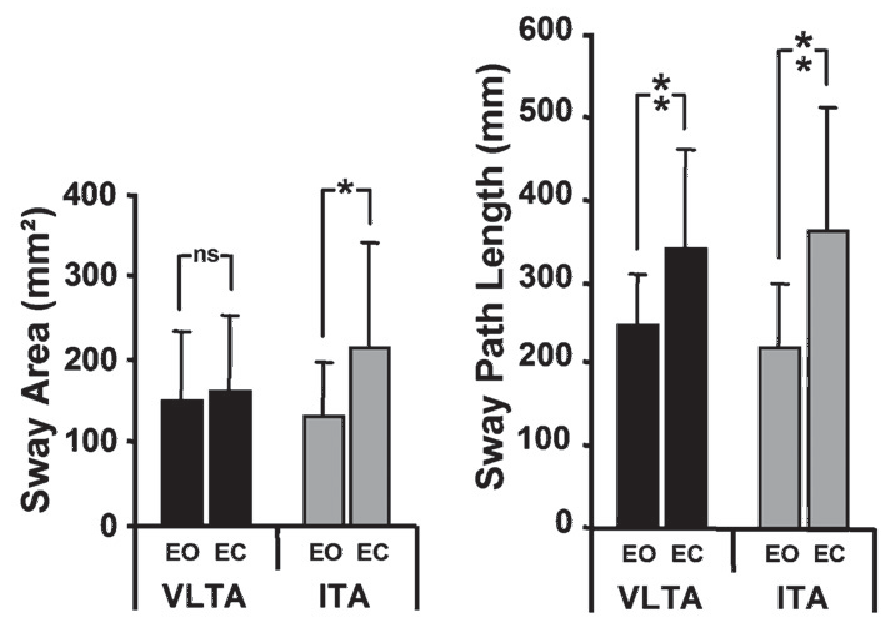

Fig. 3. Control condition: differences of balance performances (sway area and sway path length) between eyes-open (EO) and eyes-closed (EC) tests. ${ }^{*} p<0.01$, ${ }^{* *} p<0.001$, significant difference between EO and EC tests.
$[7,9,10,23,25]$, we suggest that in this condition, state anxiety may influence vestibular input and/or somatosensory input. Moreover, the effects of state anxiety on static balance control do not seem to be dependent on whether visual input is present for VLTA subjects. In contrast, static balance control of ITA subjects may not be influenced by the anxiogenic condition when vision is absent. Supplementary statistical analyses carried out in the control condition (see Fig. 3) highlight some specificities in the way balance is regulated when vision is absent. Comparisons between eyes-open and eyes-closed performances have shown that SPL increases in both groups, while SA increases in the ITA group only. In agreement with previous studies, VLTA subjects tended to compensate for the lack of visual input with an ankle stiffness strategy [5]. This somatosensory regulation of balance, which increases the SPL parameter, allows the SA parameter to be maintained when the eyes are closed [4,27]. In contrast, in this condition, the ITA subjects do not seem to use a strategy like this to compensate for the absence of visual input. These discrepancies could be explained by a visual dependence phenomenon. Studies have shown that anxious patients were visually dependent in balance performance $[28,29]$. Moreover, it has been also suggested that ITA subjects have a greater visual dependence than VLTA subjects while performing a perceptual-motor task [30]. In agreement with this concept of visual dependence, our own results in the control condition show a significant SA increase in eyes-closed compared to eyes-open performances. We suggest that the lack of visual input could disrupt the precision of static balance control in ITA subjects more than in VLTA subjects. Given this potential visual dependence, we have hypothesized that the ITA subjects could be perturbed by the EC test even in the control condition. In addition, the state anxiety induced remains moderate and could not be sufficient to cause additional perturbation. The lack of increase in SA and SPL parameters between control and 
anxiogenic conditions in the eyes-closed test in ITA subjects could suggest that moderate state-anxiety does not disturb the ability to use vestibular input and/or somatosensory input. The main discrepancy between VLTA and ITA groups is that VLTA subjects are less visually dependent than ITA subjects, and therefore more sensitive to the effects of moderate state anxiety in the EC test. Future studies should be aimed at investigating higher level of state anxiety.

\section{Conclusion}

Our results provide further evidence that state and trait anxiety could affect sensory inputs involved in balance performance. When vision is available, whatever the trait anxiety level, state anxiety could modify the processing or integration of visual, vestibular and/or somatosensory inputs. When vision is absent, the precision of static balance control in individuals with an intermediate level of trait anxiety, characterized by a higher visual dependence, is altered. Furthermore, moderate state anxiety appears to disturb the ability of individuals with a lower trait anxiety, but not that of individuals with intermediate trait anxiety, to use vestibular and/or somatosensory inputs. These results confirm the interdependence of trait anxiety, state anxiety and static balance control. New clinical tools should therefore incorporate psychological state, including intrinsic predispositions, in order to obtain a comprehensive assessment of balance control.

\section{Acknowledgements}

The authors express sincere appreciation to the referees for their helpful comments and suggestions on an earlier version of the manuscript.

\section{Conflict of interest}

There are no conflicts of interest.

\section{References}

[1] Visser JE, Carpenter MG, van der Kooij H, Bloem BR. The clinical utility of posturography. Clin Neurophysiol 2008;119:2424-36.

[2] Era P, Heikkinen E. Postural sway during standing and unexpected disturbance of balance in random samples of men of different ages. J Gerontol 1985;40:287-95.

[3] Kapoula Z, Le TT. Effects of distance and gaze position on postural stability in young and old subjects. Exp Brain Res 2006;173:438-45.

[4] Le TT, Kapoula Z. Role of ocular convergence in the Romberg quotient. Gait Posture 2008;27:493-500.

[5] Fitzpatrick R, McCloskey DI. Proprioceptive, visual and vestibular thresholds for the perception of sway during standing in humans. J Physio 1994:478.1:173-86.
[6] Adkin AL, Frank JS, Carpenter MG, Peysar GW. Postural control is scaled to level of postural threat. Gait Posture 2000;12:87-93.

[7] Bolmont B, Gangloff P, Vouriot A, Perrin PP. Mood states and anxiety influence abilities to maintain balance control in healthy human subjects. Neurosci Lett 2002;329:96-100.

[8] Carpenter MG, Frank JS, Silcher CP, Peysar GW. The influence of postural threat on the control of upright stance. Exp Brain Res 2001;138:210-8.

[9] Ohno H, Wada M, Saitoh J, Sunaga N, Nagai M. The effect of anxiety on postural control in humans depends on visual information processing. Neurosci Lett 2004;364:37-9.

[10] Wada M, Sunaga N, Nagai M. Anxiety affects the postural sway of the anteroposterior axis in college students. Neurosci Lett 2001;302:157-9.

[11] Furman JM, Jacob RG. A clinical taxonomy of dizziness and anxiety in the otoneurological setting. J Anxiety Disord 2001;15:9-26.

[12] Sklare DA, Konrad HR, Maser JD, Jacob RG. Special issue on the interface of balance disorders and anxiety: an introduction and overview. J Anxiety Disord 2001;15:1-7.

[13] Kogan E, Lidor R, Bart O, Bar-Haim Y, Mintz M. Comorbidity between balance and anxiety disorders: verification in a normal population. J Psychol 2008;142:601-13.

[14] Yardley L, Owen N, Nazareth I, Luxon L. Prevalence and presentation of dizziness in a general practice community sample of working age people. Br J Gen Pract 1998;48:1131-5.

[15] Brown LA, Polych MA, Doan JB. The effect of anxiety on the regulation of upright standing among younger and older adults. Gait Posture 2006;24: 397-405.

[16] Carpenter MG, Frank JS, Silcher CP. Surface height effects on postural control: a hypothesis for a stiffness strategy for stance. J Vestib Res 1999;9:277-86.

[17] Hainaut JP, Bolmont B. Moderate state-anxiety differently modulates visual and auditory response times in normal- and very low trait-anxiety subjects. Neurosci Lett 2006;395:129-32.

[18] Hainaut JP, Monfort V, Bolmont B. Trait-anxiety dependence of movement time performance in a bimodal choice task in subjects exposed to moderate anxiogenic conditions. Neurosci Lett 2006:409:196-9.

[19] Spielberger CD. Anxiety: current trends in theory and research. New York: Academic Press; 1972.

[20] Leite JR, Seabra Mde L, Sartori VA, Andreatini R. The video-recorded Stroop Color-Word Test as a new model of experimentally-induced anxiety. Prog Neuropsychopharmacol Biol Psychiatry 1999;23:809-22.

[21] Teixeira-Silva F, Prado GB, Ribeiro LC, Leite JR. The anxiogenic video-recorded Stroop Color-Word Test: psychological and physiological alterations and effects of diazepam. Physiol Behav 2004;82:215-30.

[22] Spielberger CD, Gorsuch RL, Lushene R, Vagg PR, Jacobs GA. Manual for the State-Trait Anxiety Inventory (Form Y) (Self-Evaluation Questionnaire). Palo Alto (CA): Consulting Psychologists Press; 1983.

[23] Balaban CD, Porter JD. Neuroanatomic substrates for vestibulo-autonomic interactions. J Vestib Res 1998;8:7-16.

[24] Balaban CD, Thayer JF. Neurological bases for balance-anxiety links. J Anxiety Disord 2001;15:53-79.

[25] Jacob RG, Furman JMR, Clark DB, Durrant JD, Balaban CD. Psychogenic dizziness. In: Sharp, Barber, editors. The Vestibulo-ocular reflex and vertigo. New York: Raven Press; 1993.

[26] Hoehn-Saric R, Hazlett RL, Pourmotabbed T, McLeod DR. Does muscle tension reflect arousal? Relationship between electromyographic and electroencephalographic recordings. Psychiatry Res 1997;71:49-55.

[27] Ouaknine M. Posture and vision. In: 4th symposium of CEOPS; 2006.p. 18-22.

[28] Jacob RG, Furman JM, Durrant JD, Turner SM. Panic, agoraphobia, and vestibular dysfunction. Am J Psychiatry 1996;153:503-12.

[29] Yardley L, Britton J, Lear S, Bird J, Luxon LM. Relationship between balance system function and agoraphobic avoidance. Behav Res Ther 1995;33:435-9.

[30] Viaud-Delmon I, Ivanenko YP, Berthoz A, Jouvent R. Adaptation as a sensoria profile in trait anxiety: a study with virtual reality. J Anxiety Disord 2000;14:583-601. 